\title{
Research Paper \\ Evaluating Specivity, Sensitivity, Positive and Negative Predictive Values of CA125 for Diagnosing Ovarian Cancer
}

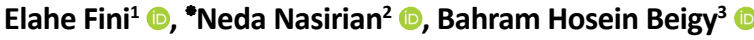

1. Department of General Practitioner, School of Medical Shahid Babaee, Qazvin University of Medical Science, Qazvin, Iran.

2. Department of Pathobiology, Shahid Babaei School of Medicine, Qazvin University of Medical Sciences, Qazvin, Iran.

3. Department of Parasitology, Shahid Babaei School of Medicine, Qazvin University of Medical Sciences, Qazvin, Iran.

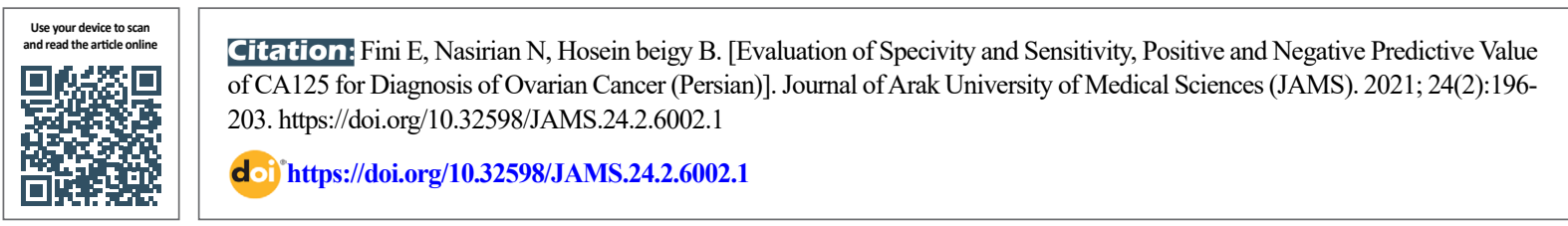

\section{(i) (8)}

Article Info:

Received: 11 Oct 2019

Accepted: 19 Apr 2021

Available Online: 01 Jun 2021

Keywords:

Ovarian cancer, CA125, Tumor marker Diagnostic value

\section{A B STRACT}

Background and Aim Ovarian cancer is among the most common cancers in women worldwide. CA125 is the most frequent biomarker used in the screening for ovarian cancer. CA125 has no high sensitivity and specificity as a screening test in the medical community; however, because of being simple and noninvasive, it is almost always requested for evaluation and ruling out cancer. It plays an important role in the treatment and post-treatment process, the prediction of prognosis, and the relapse of the disease. The present study aimed to determine the relationship between a high level of CA125 tumor marker and ovarian cancer by detecting spesivity, sensivity, positive and negative predictive values.

Methods \& Materials In this cross-sectional study, all cases undergoing CA125 test in Velayat Hospital in 2017-1028 were evaluated for having ovarian cancer. In addition, the CA125 level was compared between healthy individuals and patients with ovarian cancer. Finally, the obtained data were analyzed using SPSS. Ethical Considerations The present study was approved by the Qazvin University of Medical Sciences (Ethics Code: IR.QUMS.REC.1396.316).

Results In this study, $35.3 \%$ of the study participants received a definite diagnosis of ovarian cancer. Generally, CA125 values were negative in $41.8 \%$ and positive in. $58.2 \%$ of the study subjects. The sensitivity of the test was measured as $80.1 \%$, the specivity as $53.6 \%$, the positive predictive value equaled $48.4 \%$, and the negative predictive value was measured as $83 \%$. There was a significant relationship between age and the presence of ovarian cancer, and serum CA125 levels.

Conclusion The present study suggested that age and the serum level of CA125 were statistically significant. Finally, CA125 levels were significantly related to ovarian cancer. It provided moderate specivity and specivity as well as low positive predictive value and high negative predictive value as a tumor marker; it is valuable for ruling out of tumor but not appropriate as a screening test.

\section{* Corresponding Author: \\ Neda Nasirian, PhD.}

Address: Department of Pathobiology, Shahid Babaei School of Medicine, Qazvin University of Medical Sciences, Qazvin, Iran.

Tel: +98 (912) 6826675

E-mail: qums@gmail.com 


\section{Extended Abstract}

\section{Introduction}

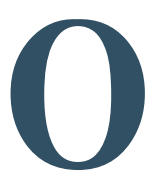

varian carcinoma is the most common type of ovarian cancer [1]. According to global statistics in 2012, ovarian cancer was the seventh most frequent type of cancer and the eighth leading cause of cancer-induced death in women [2]. CA125 is a member of the glycoprotein family and as a biomarker in the body, i.e., the most widely used biomarker in ovarian cancer screening [3, 4]. However, according to studies, CA125 is not very sensitive and specific to be used as a screening test in the medical community. This is because this biomarker rises in other cancers, such as the endometrium, breast, lung, etc., as well as physiological conditions such as pregnancy and menstruation. Moreover, this biomarker is not necessarily elevated in every disease with ovarian cancer [9]. It is simple and non-invasive; thus, this test is almost always performed and plays an important role in identifying women with ovarian mass in ultrasound as well as the treatment process and after treatment concerning determining the prognosis and recurrence of the disease. This study explored the relationship between high CA125 and the presence of ovarian cancer and the practical value of measuring CA125 as a screening test to determine the sensitivity, specificity, and positive and negative predictive value of this test in Iranian patients.

\section{Materials and Methods}

This cross-sectional study was performed epidemiologically and descriptively with analytical aspects; all CA125 tests performed in 2018-2019 in Hospitals affiliated with the university referral center for this test in Qazvin Province, Iran, were examined for ovarian cancer. In addition, CA125 levels were compared between healthy individuals and patients with ovarian cancer.

CA125 was measured using the Electrochemiluminescence (ECL) method, i.e., the most accurate method of performing this test. Besides, it was performed by an experienced individual by a Roch kit in a single laboratory. The normal values of CA125 were considered to range between 0-35 U/mL.

Next, a checklist was prepared in which patients' data, including age, complaints, diagnosis, and CA125 were recorded. Finally, the obtained data were analyzed in SPSS.

Descriptive findings were also extracted. T-test was used to compare the mean age and the relationship between a positive test and the presence of ovarian cancer in the study subjects. Probability values of below 0.05 were considered significant. Then, according to the collected data, sensitivity (true positive test/total patients), negative predictive value, specificity (true negative test/total healthy individuals), positive predictive value (true positive test/total positive test cases), and negative predictive value (true negative test cases/total negative test cases) were measured. All cases in which the patient was unavailable after the test and it was unclear whether or not they had ovarian cancer were excluded from the study.

\section{Results}

In total, 400 patients for whom the CA125 test was performed in Qazvin hospital laboratory between 2015 and 2019 were included in this study. Of them, 141(35.3\%) cases had a definite diagnosis of ovarian cancer, while in the remaining 259(64.7\%) subjects, ovarian cancer was ruled out with a pathological diagnosis. In general, CA125 values were negative in $167(41.8 \%)$ cases and positive in 233(58.2\%) subjects.

The Mean \pm SD age of study participants was $57.97 \pm 17.3$ years. The youngest sample was 17 years old and the oldest was 98 years old. The Mean \pm SD age in the group with a diagnosis of ovarian cancer was $60.14 \pm 16.6$ years and in the healthy group in terms of ovarian cancer, it was $54.94 \pm 17$ years. The relevant results of the Independent Samples ttest indicated that the relationship between age and serum levels of CA125 was significant $(\mathrm{P}=0.003)$. The relationship between CA125 levels and the diagnosis of ovarian cancer was evaluated. The relationship between these two parameters is summarized in Table 1 . The Chi-squared test data revealed that the levels of CA125 were significantly correlated with ovarian cancer $(\mathrm{P}<0.0001)$. Finally, the parameters related to the diagnostic value of CA125 measurement were calculated to evaluate ovarian cancer. The present research results highlighted that the sensitivity of the test was equal to $80.1 \%$, its specificity equaled $53.6 \%$, the test's positive predictive value and negative predictive value were measured to be $48.4 \%$ and $83 \%$, respectively.

Sensitivity: True positive test/total patients: $141 / 113$

Feature: True negative test/total healthy individuals: 259/139

Positive predictive value: True positive tests/Total positive test cases: $223 / 113$

Negative predictive value: True negative test items/Total negative test items: 167139 
Table 1. The relationship between high test values and diagnosing ovarian cancer

\begin{tabular}{cccc}
\hline \multirow{2}{*}{ Characteristic } & \multicolumn{3}{c}{ No. (\%) } \\
\cline { 2 - 4 } & Positive Concerning CA125 Level & Negative Concerning CA125 Level & Total \\
\hline Positive concerning ovarian cancer & $113(48.5)$ & $28(16.8)$ & $141(35.3)$ \\
Negative concerning ovarian cancer & $120(51.5)$ & $139(83.2)$ & $259(64.7)$ \\
\hline Total & $233(100)$ & $167(100)$ & $400(100)$ \\
\hline
\end{tabular}

\section{Discussion and Conclusion}

This study revealed that the CA125 levels as well as the age of the explored patients were significantly associated with presenting ovarian cancer. Furthermore, concerning the diagnostic value of CA125 in the study of ovarian cancer, test sensitivity was measured as $80.1 \%$, specificity: $53.6 \%$, positive predictive value: $48.4 \%$, and negative predictive value: $83 \%$. Various international and domestic studies have examined the association between CA125 and ovarian cancer. In this regard, Lycke et al. (2018) examined the diagnostic value of CA-125, HE4, and ROMA in determining the risk of ovarian mass malignancy in Sweden. They concluded that CA125 is superior to HE4 as a biomarker in identifying women with ovarian cancer [10].

Compared to the above-mentioned study, our research also signified that high levels of CA125, despite ovarian cancer, were significant correlated with a very low P-value; thus, our study data were consistent with those of Lycke and associates. Additionally, compared to the above study, although the positive predictive value in our study was lower, the sensitivity and specificity values and the negative predictive value of the test were almost consistent with the results of this study. The results of Yousefi et al.'s study (2014) indicated that the mean age of patients with malignant mass was significantly higher than that of the patients with benign mass [12].

Compared to their results, age, in our study, was significantly related to the presence of ovarian cancer; there was also a significant relationship between the presence of ovarian cancer and CA125 levels, i.e., consistent with the results of the study by Yousefi and colleagues. Besides, in a study conducted in 2015 by Pallavi Anand in India, several patients at Kanpur Hospital who presented with early signs of abdominal pain, infertility, etc. were screened with the CA125 biomarker test. Ovarian cancer rates have risen further, accordingly [13]. The results of other studies indicated the role of this biomarker in the early diagnosis of ovarian cancer. It also depends on the prognosis and response to treatment.

\section{Ethical Considerations}

\section{Compliance with ethical guidelines}

The present study was approved by the Thesis Council of Qazvin University of Medical Sciences (Ethics Code: IR.QUMS.REC.1396.316). The researchers adhered to all the principles of the protocols and guidelines recommended by the Helsinki Convention on Ethics in Research. Finally, to maintain confidentiality issues, the results of this study were reported without disclosing the names and identifiable details of patients.

\section{Funding}

This research did not receive any grant from funding agencies in the public, commercial, or non-profit sectors.

\section{Authors' contributions}

All authors equally contributed to preparing this article.

\section{Conflicts of interest}

The authors declared no conflicts of interest.

\section{Acknowledgements}

We would like to thank the laboratory and pathology department of Velayat Hospital for their sincere cooperation, especially with Ms. Islami, as well as Dr. Samiei and Dr. Tahvildari, who contributed to this research. 


\section{ارزيابى حساسيت، ويزّكَى، ارزش اخبارى مثبت و منفى سطح CA-125بر اي وجود سرطان تخمدان}

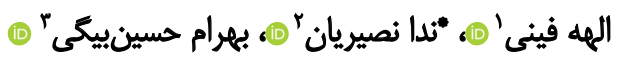

1. كروه يزشك عمومى، دانشكده هزشكى شهيد بابايى، دانشعاه علوم بزشكى قزوين، قزوين، ايران.

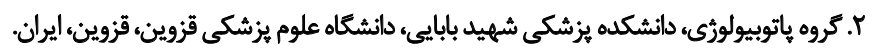

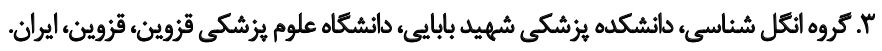

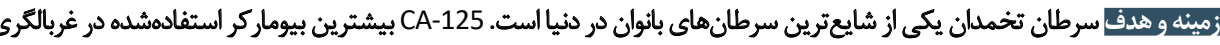

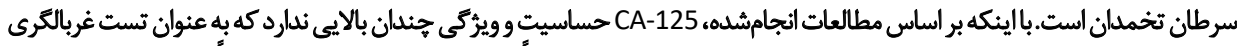

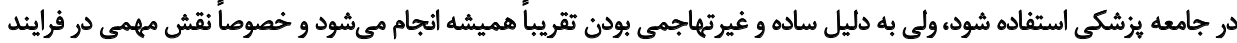

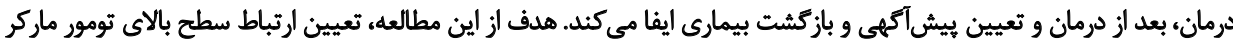

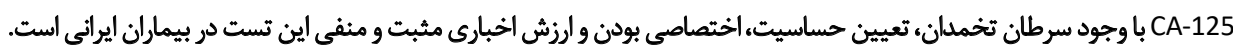

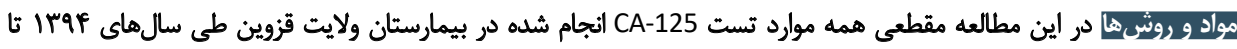

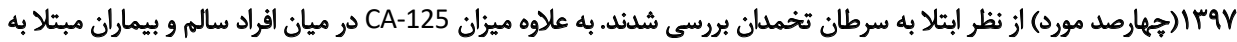

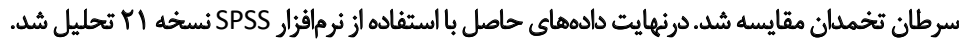

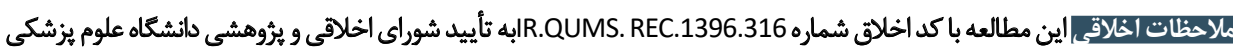
قزوين رسيده است.

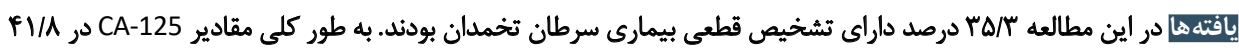

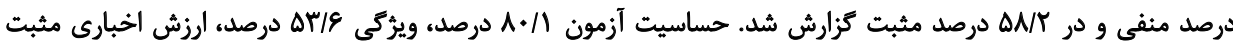

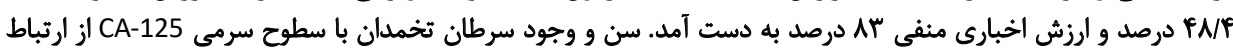
آمارى معنادار داشتند.

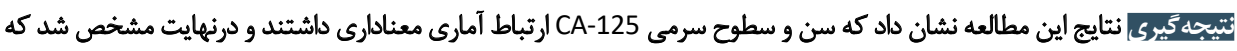

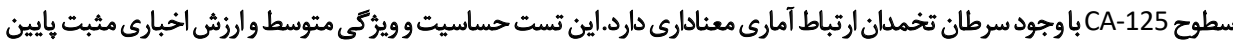

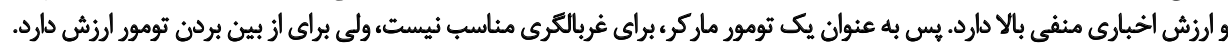

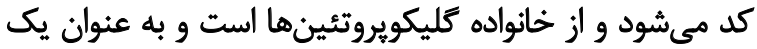

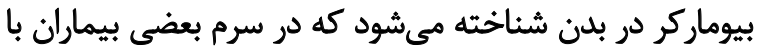

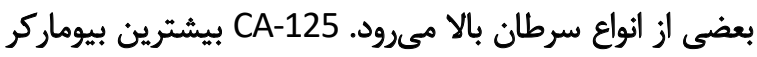

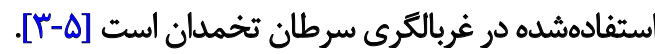

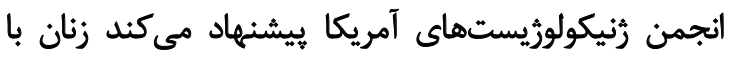

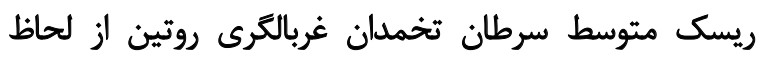

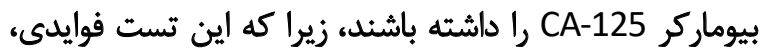

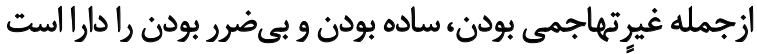

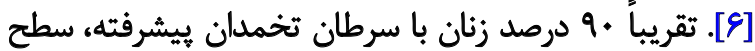

كارسينوم تخمدان رايجترين نوع سرطان تخمدان است. اغلب

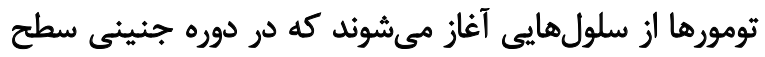

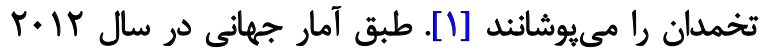

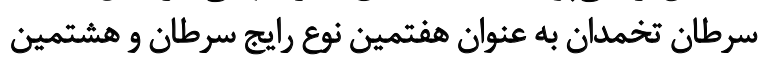

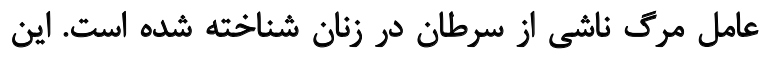

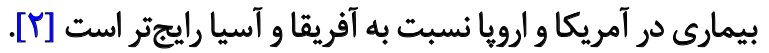
MUS16 بروتئينى است كه در بدن انسان با ثن CA-125 
ثبت شد. درنهايت دادههاى حاصل از اين مطالعه وارد نرمافزار SPSS

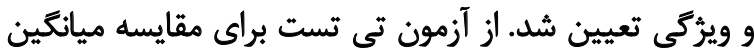

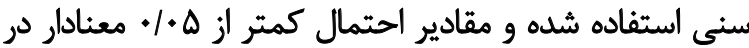

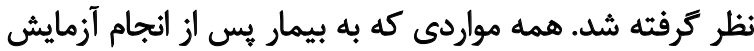

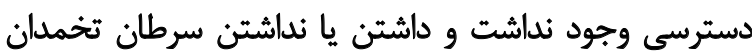
مشخص نبود، از مطالعه كنار كذاشته شد ند.

ياقتتهها

از ميان جهارصد تست انجامشده در مدت جهار سال،

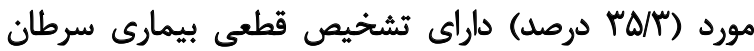

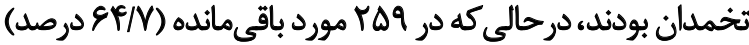

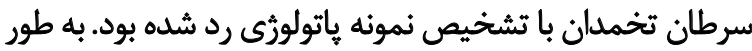

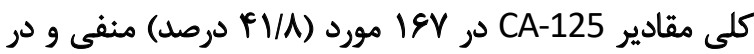

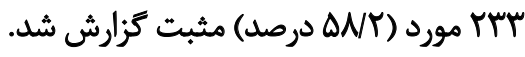

ميانكين سنى در شركت كنندكان مطالعه، DV/9V سال با

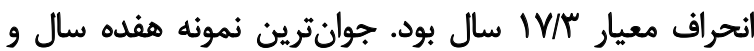
مسنترين آنها و 1 سال سن داشتند.

ميانكين سنى در كروه با تشخيص سرطان تخمدان

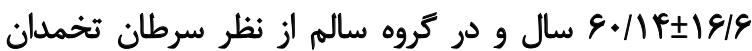
Df/Af IVIAf

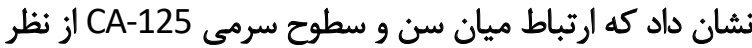

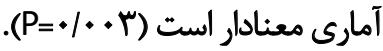

ارتباط ميان سطوح CA-125 و تشخيص سرطان تخمدان

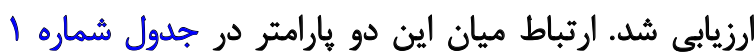

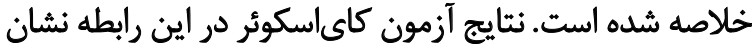

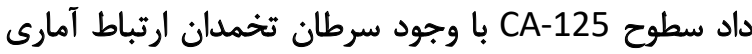

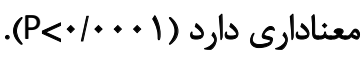

درنهايت نيز يارامترهاى مربوط به ارزش تشخيصى

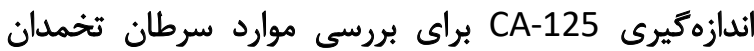

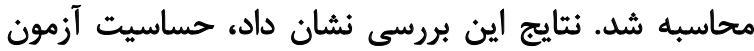

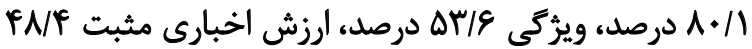

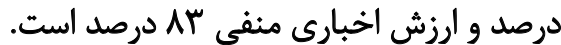

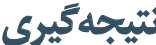

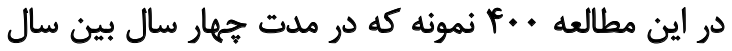

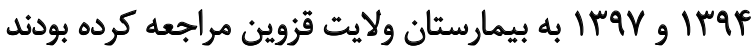
از نظر تشخيص سرطان تخمدان و سطوح بيوماركر

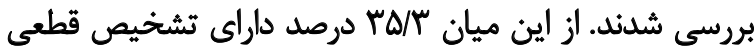

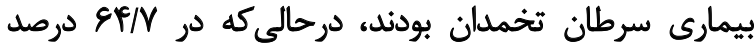

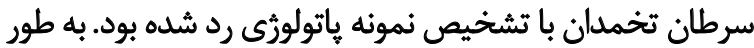

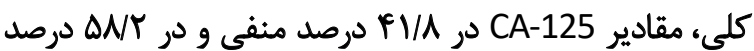

بالاى CA-125 در سرم خود دارند كه باعث مىشود نقش مفيدى إي إي

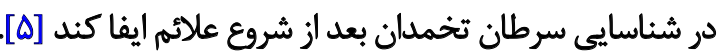
جهت تشخيص زودرس تودههاى بدخيم تخمداني

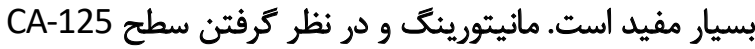

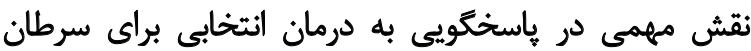

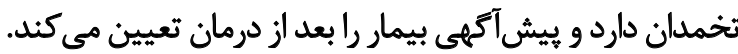

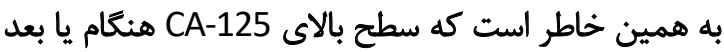

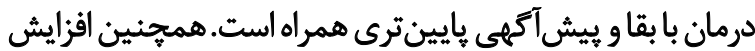

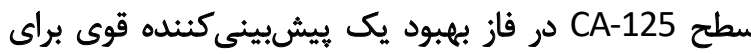

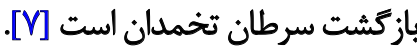
نقش تشخيص زودهنكام سرطان تخمدان هنوز ثابت نشده و

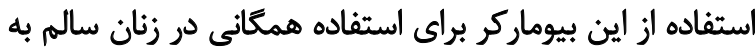

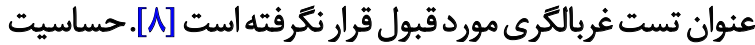

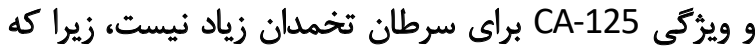

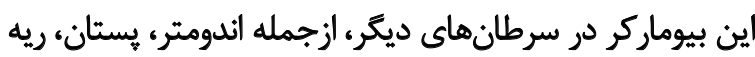

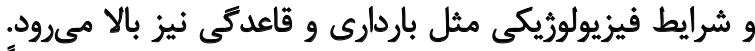

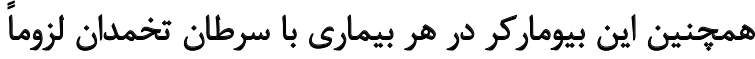

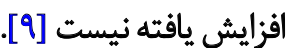

با باينكه اين تست از لحاظ حساسيت و ويزّكى آنقدر در سطح

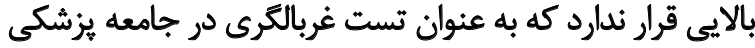

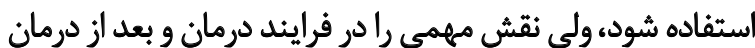

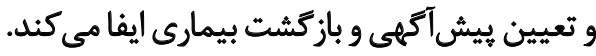
در اين تحقيق سعى شده است ارتباط بين CA-125 بالاو و

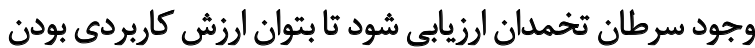

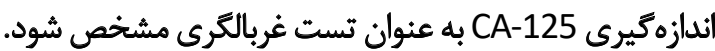

\section{مواد و روش هـا}

در اين مطالعه مقطعى كه به صورت إيدميولوريك توصيفى با

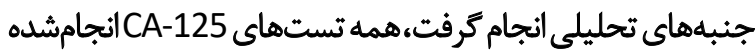

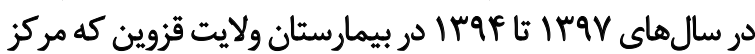

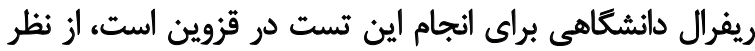

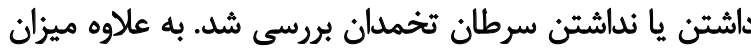

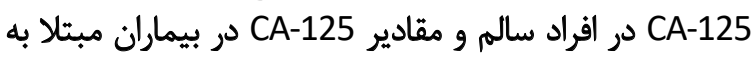

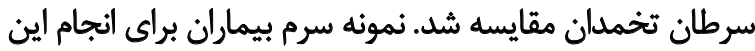

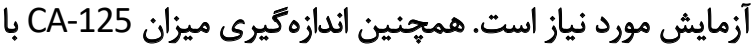

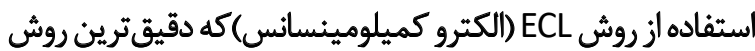

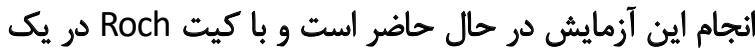

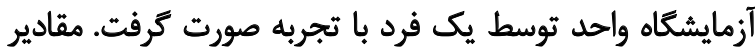

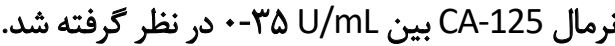
در مرحله بعد جكاليستى تهيه و اطلاعات بيماران اعم از سن،

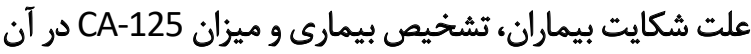


جدول ا. ارتباط ميان بالا بودن تست و تشخيص سرطان تخمدان

\begin{tabular}{|c|c|c|c|}
\hline \multicolumn{3}{|c|}{ تعداد (درصد) } & \multirow[b]{2}{*}{ مثغيرها } \\
\hline جمع (درصد) & 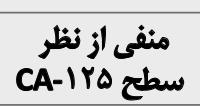 & مثح مثبت از نظر & \\
\hline$|f|(r \Delta / r)$ & $r A(|Y| A)$ & $\| T(P N A)$ & هُبت از نظظر سرطان تخملان \\
\hline$r \Delta q(\& \& / V)$ & Irq (Ar/T) & $\| r \cdot(\Delta) / \Delta)$ & منفى از نظر سرطان تخمدان \\
\hline$f \cdots(1+)$. & $\operatorname{leg}(1.0)$ & $M(1 . .0)$ & جمع \\
\hline
\end{tabular}

(n)

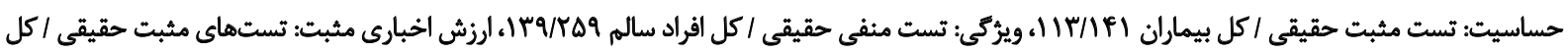

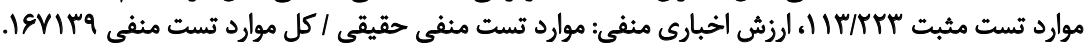

حساسيت و ويُركى و ارزش اخبارى منفى آزمون ثقريباً با نتايج اين دو مطالعه همخواني دئ دارد.

نتايج مطالعه يوسفى و همكاران در سال بوبا كه به بررسى

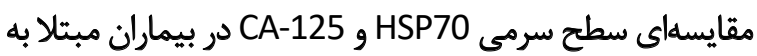
سرطان ايى تليال تخمدان و بيماران با توده خوش خيميم تخمداني

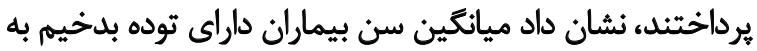

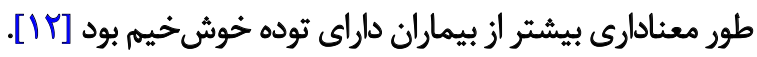
در مقايسه با نتايج مطالعه ايشان سن در مطالعه ما ارتباط

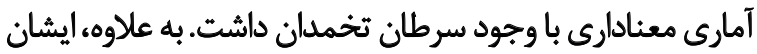

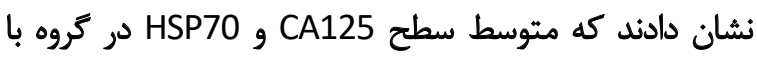

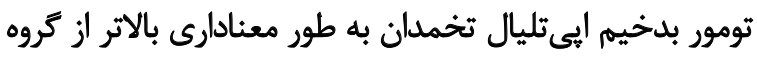

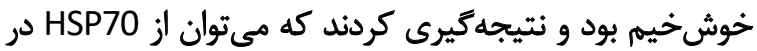

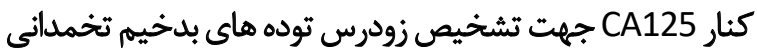
استفاده كرد.

در مقايسه با نتايج مطالعه ايشان، نتايج مطالعه ما نيز بيانكر

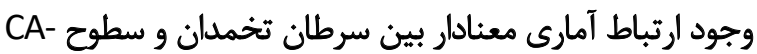

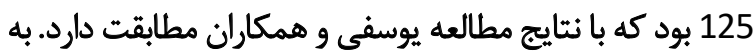

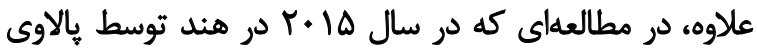

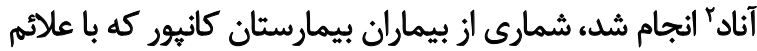

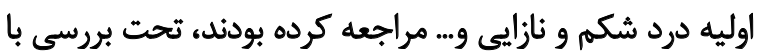

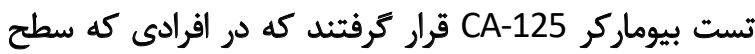
اين فاكتور بالا بوده است، آمار سرطان تخمدان افزايش بيشترى

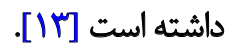

نتايج مطالعه يالاوى آناد و همكاران كه بيانكر ارزش بالاي

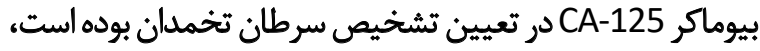
همراستا با نتّايج مطالعه ما است.

همجنين نتايج مطالعه عرب و همكاران در سال •وسار كه تأثير

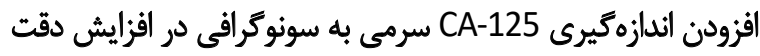

مثبت كزارش شد. نتايج آناليز تحليلى در اين مورد نشان داد سن

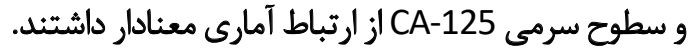

درنهايت مشخص شد كه سطوح CA-125 با وجود سرطان

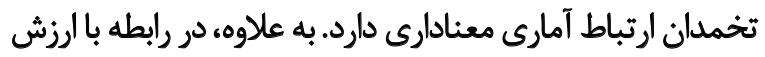

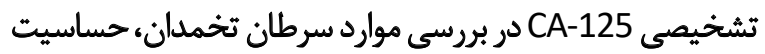

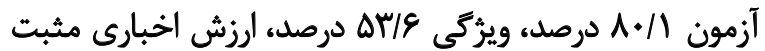

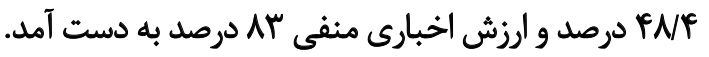
مطالعات مختلفى در ايران و ساير نقاط جهان ارتباط ميان

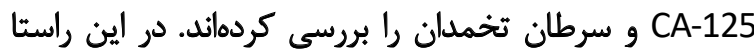

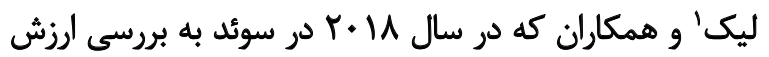

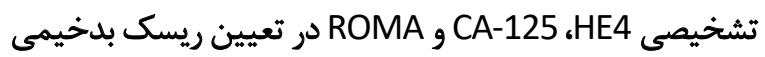

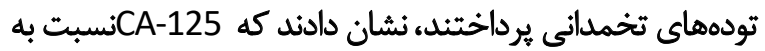

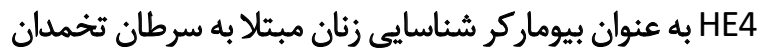

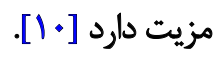

در مطالعهاى مشابه، جعفرى شبيرى و همكاران در سال

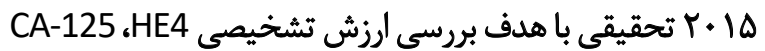

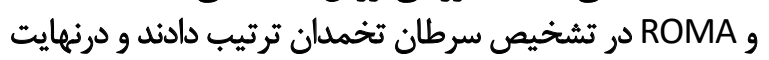

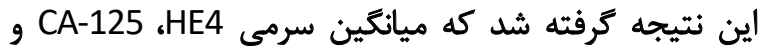

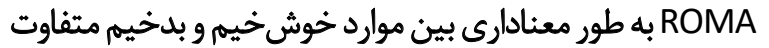

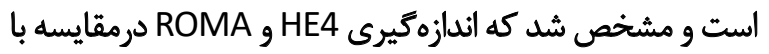

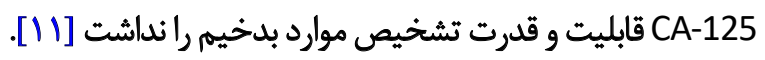

در مقايسه با دو مطالعه فوق اكرجه در مطالعه حاضر تنهها

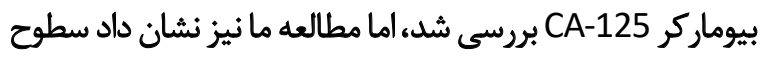

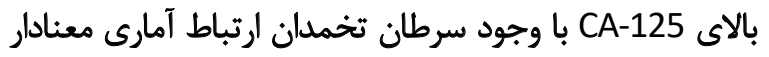

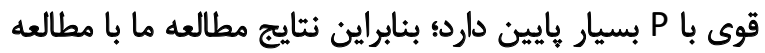

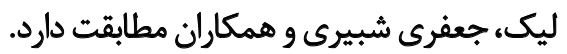

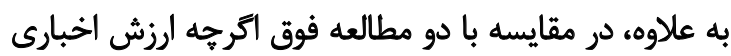

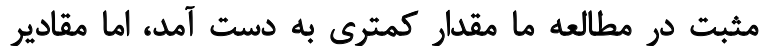


دNAf درصد و ارزش اخبارى منفى سA درصد به دست آمد. نتايج اين مطالعه نشان داد سن و سطوح سرمى

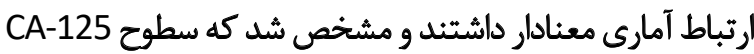
باوجود سرطان تخمدان ارتباط آمارى معنادارى دارد. آين تست

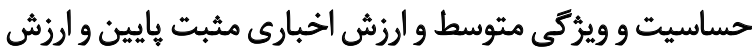

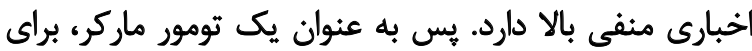
غربالكرى مناسب نيست، ولى براي از بين بردن تومور ارزش دارد. باري

مالاحظات اخلاقى

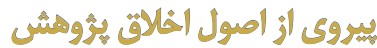

محققين به همه اصول يروتكل هاو دستور العمل هاى توصيهشده

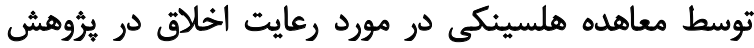

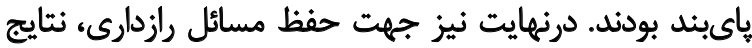

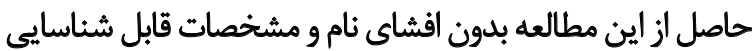

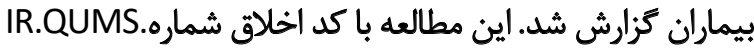

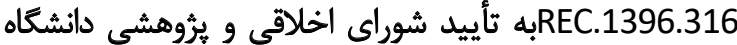

علوم يزشكى قزوين رسيده است.

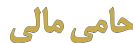

اين تحقيق هيج كونه كمك مالى از سازمان هاي تأمين مالى در

بخشهاى عمومى ، تجارى يا غير انتفاعى دريافت نكردي آنسازي

\section{مشار كت نويسند كان}

تمام نويسندكان در طراحى، اجرا و نكارش همه بخشهاى ئروهش حاضر مشاركت داشتهاند.

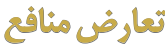

بنابر اظهار نويسندكان اين مقاله تعارض منافع ندارد.

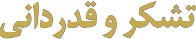

از بخش آزمايشكاه بيمارستان ولايت براى همكارى

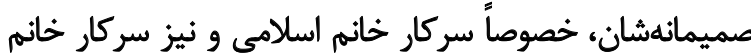

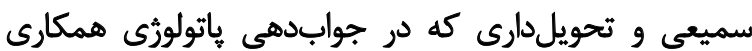
داشتهاند نهايت تشكر را داريم.
تشخيص بدخيمى قبل از جراحى در بيماران توده تخمدانى رابررسى

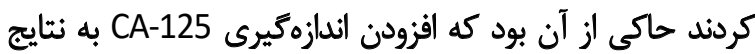

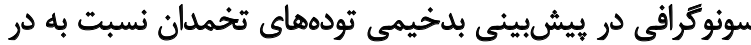

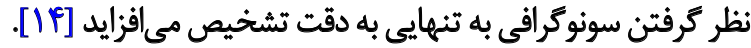

نتايج كلى اين مطالعه نيز با نتايج مطالعه ما همخيوانى دارد.

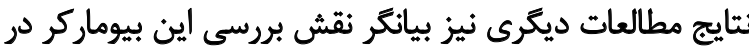
تشخيص زودرس سرطان تخمدان است.

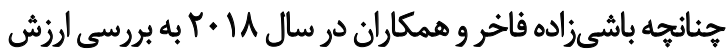

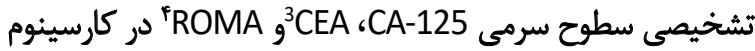
سروز تخمدان با درجه بدخيمى يايين يرداختند، نشان سيمان دادئد،

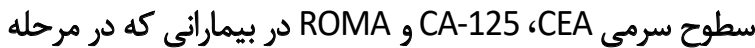

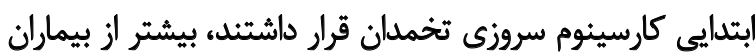

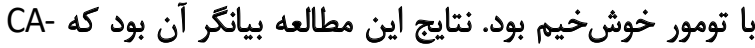
مى CEA , 125 ، ROMA

زودهنگام كارسينوم سروزى تخمدان در نظر كرفته شوند [ [ 1 ]. اما نتايج مطالعات حاكى از آن است كه بيوماركر CA-125

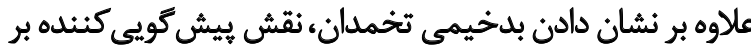
بيش آتحهى و ياسخ به درمان نيز است.

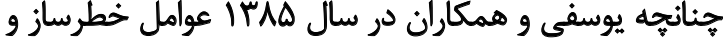

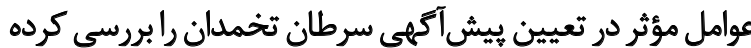

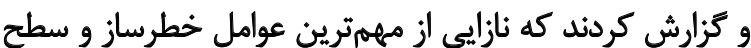

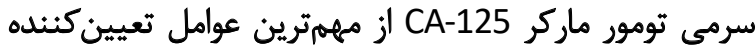
بيش آكمهى سرطان تخمدان بودند [19].

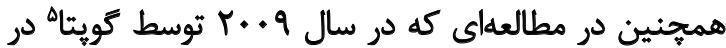

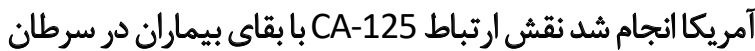

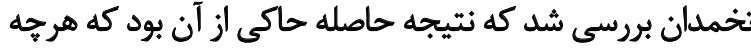

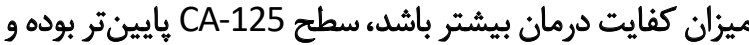

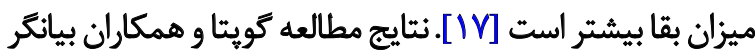

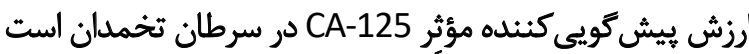
كه با نتايج مطالعه ما تقريباً همراستا است

\section{نتيجليكيرى}

نتايج اين مطالعه نشان داد كه سن و سطوح سرمى

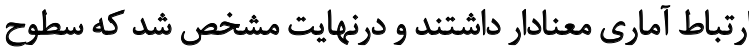

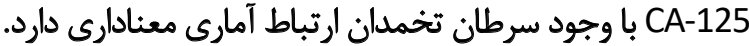

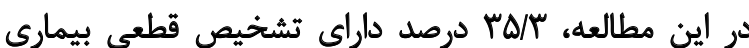

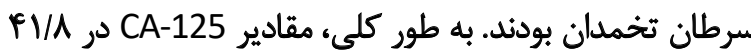

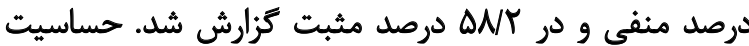

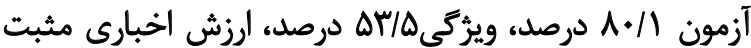

3.Carcino Emberionic Antigen

4. Risk of Ovarian Malignancy Algoritm

5. Gupta 


\section{Refrences}

[1] Noori-Daloii MR, Rashvand Z. [Molecular genetics and gene therapy in ovarian cancer (Persian)]. Horizon Med Sci . 2010; 16(3):5-19. http:// hms.gmu.ac.ir/article-1-959-en.html

[2] Marjani M. [Epidemiology and risk factors of ovarian cancer (Persian)]. Nat Assoc Iran Gyneocol Obstet. 2010; 5(3):67-73. http://www.naigo.ir/ fa/article/45

[3] Chavoshi S, Heidari-keshel S, Rezaee Tavirani M, Ebrahimi M, Etedali A, Raessodati R, et al. [Tumor markers at a glance (Persian)]. J Iran llam Univ Med Sci. 2013; 21(6):143-59. http://sjimu.medilam.ac.ir/article1-1480-fa.html

[4] Sundar S, Neal RD, Kehoe S. Diagnosis of ovarian cancer. BMJ. 2015; 351:h4443. [DOI:10.1136/bmj.h4443]

[5] Nolen BM, Lokshin AE. Biomarker testing for ovarian cancer: clinical utility of multiplex assays. Mol Diagn Ther J. 2013; 17(3):139-46. [DOI:10.1007/s40291-013-0027-6][PMID][PMCID]

[6] Duraisamy S, Ramasamy S, Kharbanda S, Kufe D. Distinct evolution of the human carcinoma-associated transmembrane mucins, MUC1, MUC4 AND MUC16. Gene J May. 2012; 373:28-34. [DOI:10.1016/j. gene.2005.12.021][PMID]

[7] Suh KS, Park SW, Castro A, Patel H, Blake P, Goy A. Ovarian cancer biomarkers for molecular biosensors and translational medicine. Expert Rev Mol Diagn. 2010; 10(8):1069-83. [DOI:10.1586/erm.10.87] [PMID]

[8] Bast Jr RC, Klug TL, St John E, Jenison E, Niloff JM, Lazarus H, et al. A radioimmunoassay using a monoclonal antibody to monitor the course of epithelial ovarian cancer. N Engl J Med. 1983; 309(15):883-7. [DOI:10.1056/NEJM198310133091503] [PMID]

[9] Göcze P, Vahrson H. [Ovarian carcinoma antigen (CA 125) and ovarian cancer (clinical follow-up and prognostic studies) (Hu)]. Orv Hetil. 1993; 134(17):915-8. [PMID]

[10] Lycke M, Kristjansdottir B, Sundfeldt K. A multicenter clini(cal trial validating the performance of HE4, CA125, risk of ovarian malignancy algorithm and risk of malignancy index. Gynecol Oncol. 2018; 151(1):159-65. [DOI:10.1016/j.ygyno.2018.08.025] [PMID]

[11] Jafari Shobeiri M, Parizad Nasirkandy M, Nazari F, Ouladsahebmadarek E, Sayyah-Melli M, Mostafa Gharabaghi P, et al. Diagnostic value of HE4, CA125 and risk of ovarian malignancy algorithm in detecting ovarian cancer. Int J Womens Health Reprod Sci. 2015; 3:208-11. [DOI:10.15296/ ijwhr.2015.43]

[12] Yousefi Z, Hasanzadeh Mofrad M, Kazemianfar Z, Ayatollahi H, Tavassoli F, Beyranvandi $\mathrm{M}$, et al. Comparison of serum levels of HSP70 and CA125 in patients with epithelial ovarian cancer and patients with benign ovarian masses. Iran J Obstet Gynecol Infertil. 2014; 17(101):1-5. [DOI:10.22038/IJOGI.2014.2913]

[13] Anand P, Choudhury V. A case study of assessment of CA-125 levels in the rural population of Kanpur. J Med Res. 2015; 1(5):139-41. http:// www.medicinearticle.com/JMR_201515_04.pdf

[14] Arab M, Maktabi M, Zham H, Kimiaei P, Davalo S. Is ovarian mass malignancy prediction accuracy increased by adding serum CA125 measurement to sonographic findings? Avicenna J Clin Med. 2012; 18(4):3740. http://sjh.umsha.ac.ir/article-1-211-en.html

[15] Bashizadeh-Fakhar $H$, Rezaie-Tavirani $M$, Zali $H$, Faraji $R$, Kazem Nejad $E$, et al. The Diagnostic value of serum CEA, CA-125, and ROMA index in low-grade serous ovarian cancer. Int J Cancer Manag. 2018; 11(5):e63397. [DOI:10.5812/ijcm.63397]
[16] Yousefi Z, Homaei F. [Risk factors and factors in determining the prognosis of ovarian cancer (Persian)]. J Med Counc Iran. 2006; 24(3):279-88. http://jmciri.ir/article-1-1080-fa.htm

[17] Gupta D, Lis CG. Role of CA125 in predicting ovarian cancer survival - a review of the epidemiological literature. J Ovarian Res. 2009; 2:13. [DOI:10.1186/1757-2215-2-13][PMID][PMCID] 